\title{
Axonal Dwindling in Early Experimental Diabetes. II. A Study of Isolated Nerve Fibres
}

\author{
J. Jakobsen \\ University Institute of Pathology and Second University Clinic of Internal Medicine, Kommunehospitalet, Aarhus, Denmark
}

Summary. In a morphometric study of isolated fibres of the common peroneal nerve in short-term diabetic rats reduced fibre calibre was observed. No segmental demyelination or remyelination was found, but the nodes of Ranvier were slightly widened and paranodal bulbi were swollen relative to fibre calibre. It is suggested that axonal dwindling is the primary event in experimental diabetes. The reduction of the myelin sheath may be a consequence of the abnormal nerve cell offshoot. The results obtained suggest that streptozotocin diabetes in the rat is a useful model for the elucidation of diabetic neuropathy.

Key words: Streptozotocin diabetes, myelinated nerve fibres, nerve degeneration, diabetic neuropathy.

In a previous study on cross sectioned nerves in early experimental diabetes a reduction of nerve fibre thickness was demonstrated [1]. The degree of fibre diminution was in good accordance with the reduction in nerve conduction known to occur in diabetic animals.

Diminution of nerve fibre thickness may result from axonal degeneration or from remyelination following segmental demyelination. Since the last mentioned alternative may escape detection in a study of cross sectioned nerves an investigation of single nerve fibres was undertaken using the animals described in the previous report.

\section{Materials and Methods}

Diabetes was induced by intravenous injection of streptozotocin in ten 19 to 20 week old Wistar rats weighing 330 to $380 \mathrm{~g}$. During the experimental period blood glucose ranged between 300 and 600 $\mathrm{mg} / 100 \mathrm{ml}$.

After a diabetes duration of four weeks the common peroneal nerves were fixed by vascular perfusion.

Ten animals matched with respect to sex, age and weight were kept as controls and treated likewise. Further details are given in the previous report [1].

After osmification for four hours one of the common peroneal nerves of each animal was macerated in glycerine, as described by Dyck [2].

The nerves were placed in a few drops of glycerine under a dissecting microscope and the epineurium and perineurium were removed. $40-50$ consecutive nerve fibres were gently isolated with two pairs of watchmaker's forcups. The fibres were transferred in a drop of glycerine to a slide and mounted under a coverglass.

The images of the fibres were projected on to a screen. Only fibres with a minimum of three internodes were accepted for study. From each rat fifteen nerve fibres were chosen at random for measurements and subsequently another five small and mediumsized fibres were sought. A total of 400 fibres from the 20 rats, comprising 1584 internodes, were examined.

The exact magnification was determined with a Leitz micrometer slide every time measurements were made. Internodal distances were measured at magnification $\times 225$ and taken to the nearest $10 \mu \mathrm{m}$. Thickness was measured using oil immersion at a magnification of $900 \times$ by averageing six equidistant values of each internode, excluding the paranodal region (Fig. 1). The gap between the adjacent myelin segments at the nodes of Ranvier was measured as the distance between the rounded ends of the compact myelin [3]. This distance, as well as the internodal thickness, was taken to the nearest $0.01 \mu \mathrm{m}$. A total of 1889 Ranvier nodes were measured. 


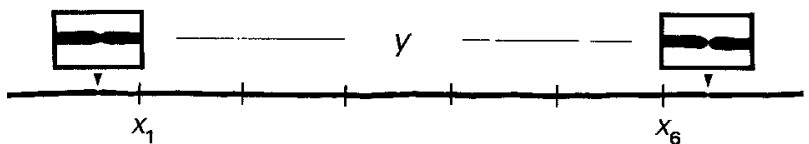

Fig. 1. Isolated nerve fibre, to demonstrate internodal length (y) and six equidistant places for measuring fibre thickness $\left(\mathrm{x}_{1-6}\right)$. Magnification $\times 50$

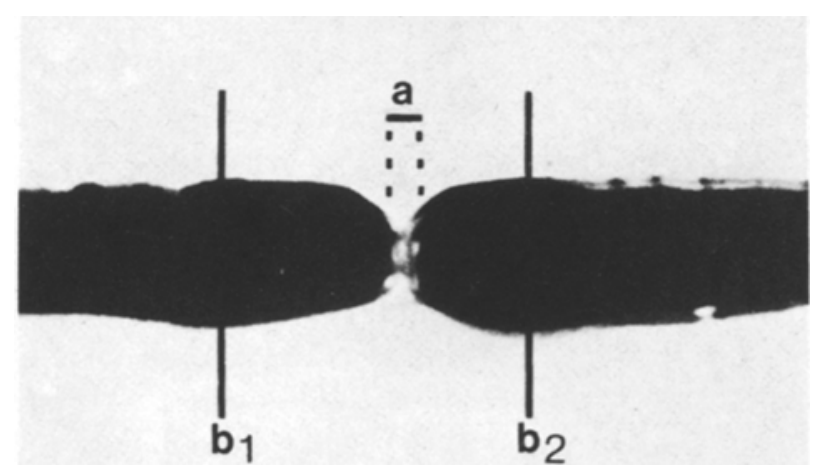

Fig. 2. Region of adjacent myelin segments, to demonstrate node width (a) and maximal thickness of neighbouring paranodal bulbi $\left(b_{1}\right.$ and $\left.b_{2}\right)$. Magnification $\times 1000$

The maximal thickness of the paranodal bulbus was measured simultaneously with the nodal gap (Fig. 2). For each internode the maximal thickness of the two paranodal bulbi were averaged.

In the fibre teasing procedure it is not possible to isolate each fibre or to obtain a representative sample of fibres of all sizes. This is not needed, however, in studies seeking information about thickness-length ratios of internodes, node width and relative paranodal dimensions or when looking for a defined maximal fibre thickness.

In the present study all fibres obtained during teasing were used for regression analyses of the relationship between length and thickness of individual internodal segments. Moreover, the five thickest fibres from each animal were selected for determination of means and standard deviation of internodal dimensions.

Internodes are graphed according to Fullerton et al. (1965), a method allowing estimation of the variation of internodal length along single nerve fibres [4].

The differences between the means of the two groups of measurements were tested for statistical significance with Student's t-test, using a 5\% limit of significance. The identity of the specimens was unknown to the technician doing the fibre-teasing and to the author doing the measurements.

\section{Results}

During fibre teasing and measurements demyelinated segments or evidence of axonal breakdown with the formation of osmiophilic droplets were not observed, either in nerves from diabetic rats or in control animals.

\section{a. Variation of Internodal Length along Single Nerve Fibres}

The variation of internodal length along single nerve fibres for all animals is shown in Figure 3. Only a very few fibres with marked variation are present.

In a single fibre of the control group four long internodes were followed by two short ones, with a thickness nearly half that of the long internodes. The following internode, part of which was present up to the point of cutting, was also thin. This type of variation may represent remyelination following segmental demyelination.

In the group of diabetic nerve fibres four were present with a single short segment, the calibres of which were reduced by $5-20 \%$ when compared to neighbouring internodes. Such segments are called short internodes in the present text.

No differences are apparent between the two groups concerning the variation of internodal length in single nerve fibres. Neither did an analysis of the variances, using a $1 \%$ limit of significance of the F-distribution, demonstrate any difference between controls and diabetic animals.

\section{b. Relation of Length and Thickness of Internodes}

Regression lines of the relationship, for each animal, between length and thickness of individual internodal segments are shown in Figure 4.

The average number of internodes available from diabetic and control rats turned out to be nearly identical $-78.6 \pm 6.8$ and $79.8 \pm 11.0$ respectively.

In the diabetic group there was a tendency to increased mean slope coefficient $(\alpha)$ of the regression lines, $0.104 \pm 0.011$ as against $0.095 \pm 0.011$ (Fig. 5). However, this difference was not statistically significant $(2 \mathrm{p}=0.088)$.

The mean correlation coefficient (r) was nearly identical in the two groups, $0.94 \pm 0.02$ in diabetics and $0.93 \pm 0.03$ in controls.

The results of the analysis of the five thickest nerve fibres for mean values of each animal is shown in Figure 6. The internodal thickness was decreased in the diabetic rats, which showed an arithmetic mean value of $11.50 \mu \mathrm{m} \pm 0.49$ compared to $12.10 \pm 0.68$ in the controls $(2 p=0.035)$. The mean value of inter- 


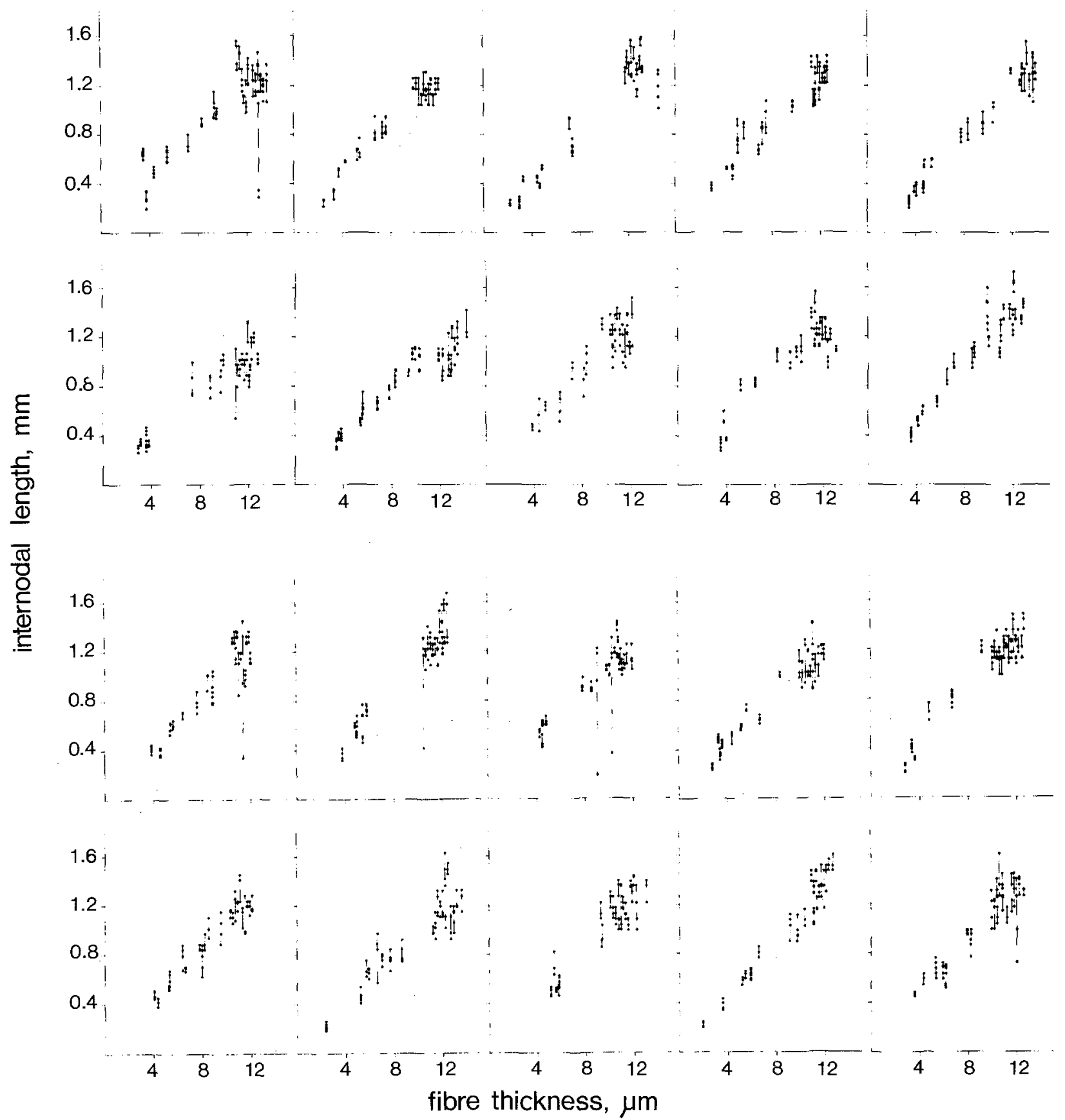

Fig. 3. Distribution of internodal lengths for fibres of different calibres in common peroneal nerves of ten controls (upper two rows) and of ten diabetic animals (lower two rows). Values for length of internodal segments of the same fibre are joined by a vertical line. The calibre of the fibre concerned is plotted as the thickest internodal segment

nodal length of each animal in the diabetic group was $1.26 \mathrm{~mm} \pm 0.10$ as against $1.25 \mathrm{~mm} \pm 0.10$ in the control group.

\section{c. Measurements of the Ranvier Node}

The measurements of all gaps between the adjacent myelin segments at the nodes of Ranvier, i. e. the node width, is given for diabetics and controls in the fre- quency-size histogram of Figure 7. The class-grouped data of all diabetic nodes has a mean value of $4.14 \mu \mathrm{m}$ $\pm 1.66(\mathrm{n}=944)$ as against $3.26 \mu \mathrm{m} \pm 1.76(\mathrm{n}=945)$ for the control nodes.

It is of special interest that no cases of extreme widening occur in the diabetic group, a fact which is also reflected in the homogeneity of the variances of the two distributions. 


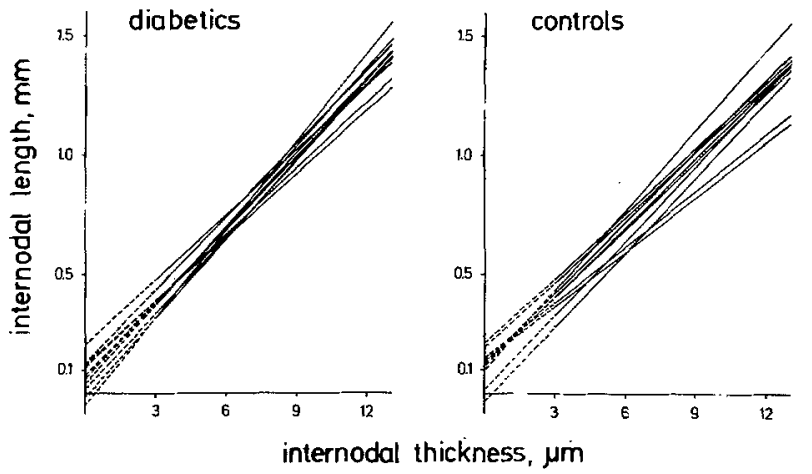

Fig. 4. Regression lines of internodal length and thickness in common peroneal nerves in each of ten controls and ten diabetic animals
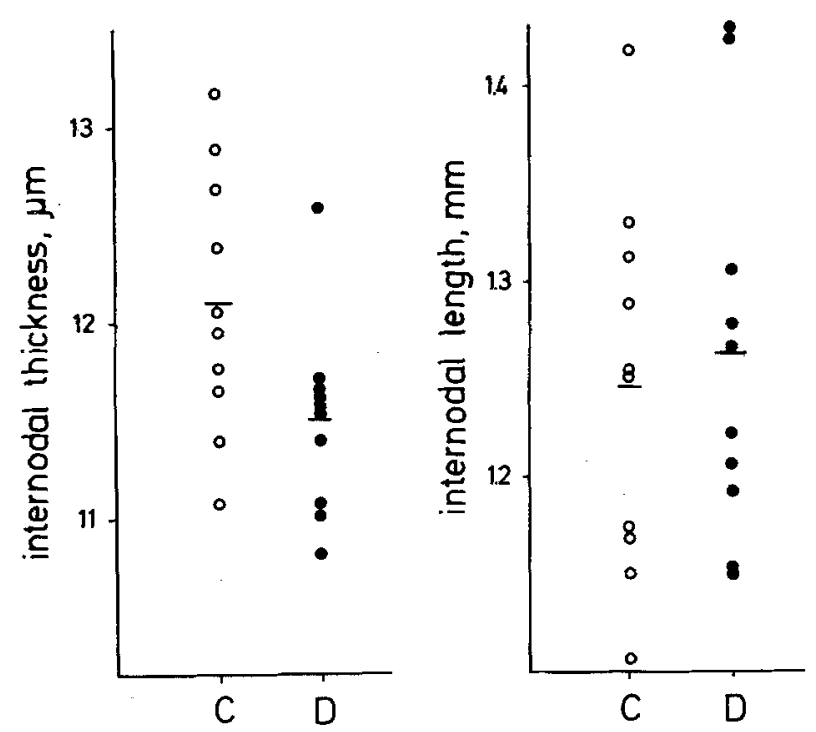

Fig. 6. Mean values of internodal thickness and length of the five thickest fibres in common peroneal nerves in each of ten controls (0) and ten diabetic animals (•)

Mean values of the nodal width calculated for each animal is shown in Figure 8. In the group of diabetics the node of Ranvier is widened by $27 \%$, a difference which is statistically significant $(2 \mathrm{p}=0.028)$.

\section{d. Measurements of the Paranodal Bulbus}

For each animal a mean maximal bulbus thickness was calculated. The values of the diabetic group did not

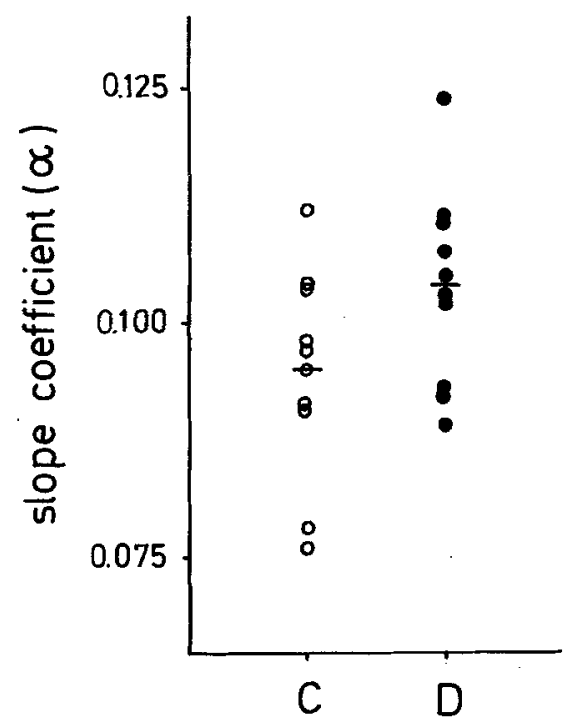

Fig. 5. Values of slope coefficients $(\alpha)$ of regression lines of internodal length and thickness in common peroneal nerves in each of ten controls $(O)$ and ten diabetic animals $(\bullet)$

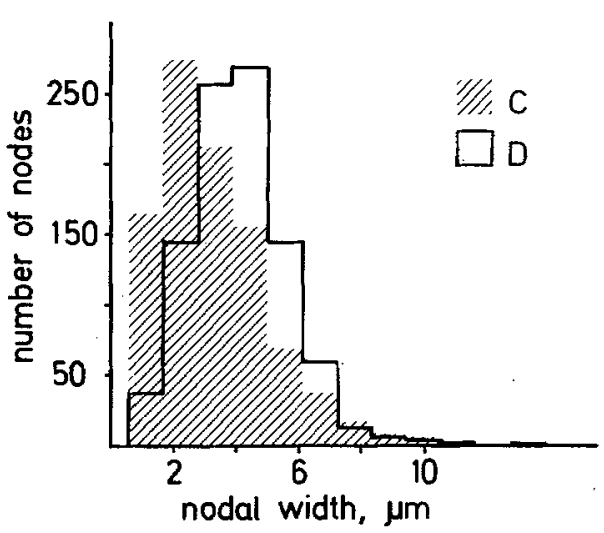

Fig. 7. Distribution of Ranvier node widths for all nodes in common peroneal nerves of controls (hatched area) and of diabetic animals (area under full-drawn line)

differ significantly from those of the controls, $8.89 \mu \mathrm{m}$ \pm 0.83 as against $8.54 \mu \mathrm{m} \pm 0.64$, respectively $(2 \mathrm{p}=$ 0.31 ).

A ratio between the bulbus thickness and the internode thickness was calculated for each internodal segment. The mean values for each animal are shown in Figure 9. An increase of relative maximal bulbus thickness is demonstrated in diabetics as compared to controls $(2 \mathrm{p}=0.010)$. 


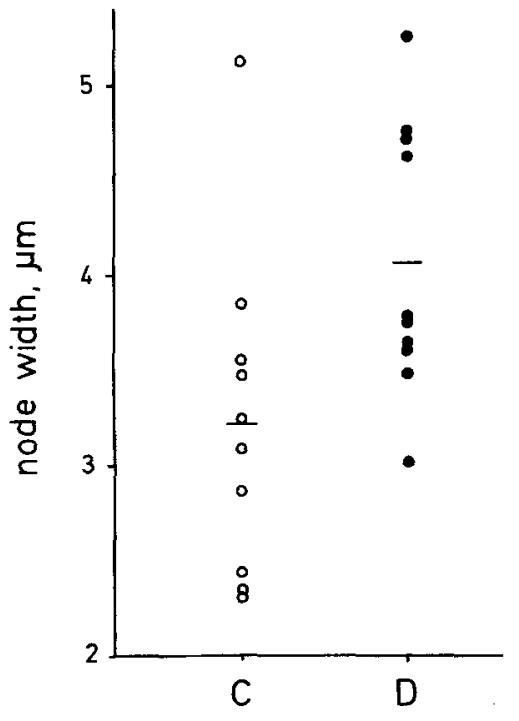

Fig. 8. Mean values of nodal width in common peroneal nerves in each of ten controls $(0)$ and ten diabetic animals $(\bullet)$

\section{Discussion}

The results reported here from a study of isolated diabetic nerve fibres have demonstrated fibre calibre diminution without the occurrence of segmental demyelination and without the thin and short segments that indicate fibres recovering from this process.

When the five largest fibres from each animal were compared in the groups of diabetics and controls, it was clear that those of the diabetic rats were thinner than those of the controls. For all isolated fibres the comparison was performed by a regression analysis of internodal length and thickness. It appeared that the average slope coefficient was higher in the diabetic group than in the control group, suggesting relatively thinner fibres in diabetic animals. However, this difference was not statistically significant.

The reason why the slope coefficient was not significantly increased may be ascribed to the fact that small fibres, as well as large fibres, were thinner [1], but also to difficulties in obtaining enough small and medium-sized fibres. As to the fibre calibre of the present study, an analysis of internodal length and thickness of a defined group of large fibres is more adequate than regression analysis of all fibres.

Signs of segmental demyelination and remyelination were not observed in this study. Two internodes from one control rat, out of a total of 798 , showed abnormal internodal length variation. Among 786 diabetic internodes, four were abnormally short segments. These findings are, of course, of no consequence.

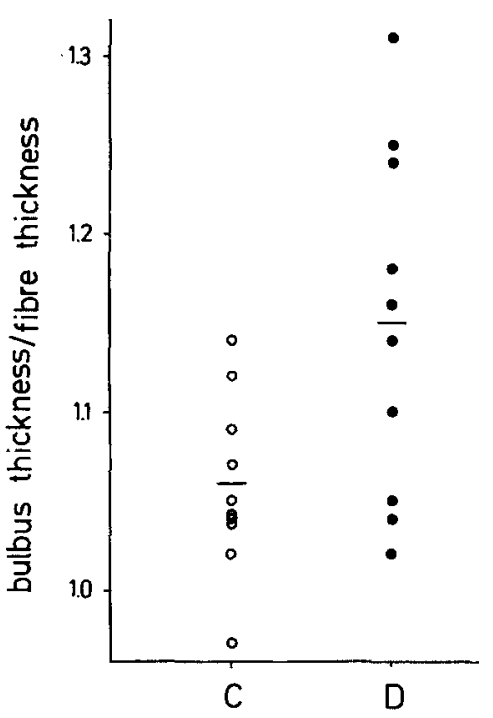

Fig. 9. Mean ratios of maximal bulbus thickness to fibre thickness in common peroneal nerves in each of ten controls $(O)$ and ten diabetic animals $(\bullet)$

The absence of segmental demyelination and remyelination is in accordance with the findings of Shar$\mathrm{ma}$ and Thomas (1974) in rats with a duration of diabetes of from 3 to 49 weeks [5].

In a quantitative study Hildebrand et al. (1968) reported an increased variance of internodal length in alloxan diabetic rats of four to six months of diabetes duration [6]. The difference between this result and that obtained in the present study and that of Sharma and Thomas (1974) is not apparent. It may be of interest to note that a quantitative study of spontaneous diabetes in Chinese hamsters reported an increased disparity of internodal length [7].

In the present study a $27 \%$ widening of the Ranvier node and paranodal swelling relative to fibre thickness was found in the diabetic rats. Such a change has been mentioned as an early sign of experimental diabetic neuropathy in other reports $[8,9]$, but quantitative studies have not been published.

At present it is not known whether this widening of the Ranvier node is associated with an enlargement of the nodal membrane at the ultrastructural level. If so, this could increase the nodal activation time, resulting in decreased conduction velocity. However, in another well known neuropathy, that of diphtheria, a similar widening of the Ranvier node is observed at the light microscropic level $[3,10]$, without changes in conduction time [10]. Furthermore, studies of single diphtheritic nerve fibres did not demonstrate electrical abnormalities of the axon membrane at the Ranvier node [11].

Another functional abnormality of diabetic 
nerves, namely the increased resistance to anoxic inactivation - the Steiness' phenomenon - which also occurs in alloxan diabetic rats may possibly be associated with the node changes demonstrated here $[12,13]$.

Two main types of nerve fibre degeneration are generally recognized, axonal degeneration and segmental demyelination, diphtheritic neuropathy being the archetype of the latter. The conspicuous finding of the present study is fibre diminution, a fact that points to axonal degeneration. Segmental demyelination was not observed, but the changes at the node of Ranvier have similarities with those observed early in diphtheritic neuropathy. Thus the structural events seem to be of mixed pathology. However, the fact that the reduction of the axon is twice that of the myelin sheath establishes experimental diabetic neuropathy as a predominantly axonal disease.

The occurrence of paranodal demyelination in another predominant axonal degeneration has been described in a study of nerve compression [14]. Central to the compressive lesion fibre calibre diminution and nodal widening were demonstrated. To explain this finding Thomas (1971) advanced the hypothesis of axonal dwindling primary to demyelination [15].

Schwann cells are controlled by the axons. This is shown in Wallerian degeneration by the myelin breakdown, which starts a few days after the interruption of the nerve in the part of the fibre distal to the transection. Contrary to this, total destruction of the myelin sheath may take place in segmental demyelination, preserving the axon. It has also been shown that retardation of axon growth retards sheath growth, while acceleration of axon growth accelerates it [16].

A sequence of events in peripheral nerves of experimental diabetes may therefore be proposed: Axonal dwindling is followed by reduction of the myelin sheath and widening of the node of Ranvier. These events may proceed into segmental demyelination and complete axonal loss.

Recently, evidence of reduced axonal flow has been presented which supports the idea of primary axonal disease in nerves of diabetic animals. In a study of Schmidt et al. (1975), on fast and slow axoplasmic flow in sciatic nerves of rats with a diabetes duration of three to four weeks, a decreased accumulation of enzymes proximal to a tie was demonstrated [17]. The impairment of the flow of protein might be responsible for axons dying back. Thereby is raised the question whether experimental diabetic neuropathy primarily affects the cell body of the neuron.

After several years of diabetes the degenerative changes in the peripheral nerves in humans are of mixed pathology $[18,19,20,21]$, although segmental demyelination is usually emphasized.
It has not yet been ascertained whether axon or schwann cell change is the primary event in diabetic ncuropathy.

Schwann cell abnormalities were stressed by Thomas and Lascelles (1965 and 66) in a study of human diabetes [19,20]. In that study, however, it was also mentioned that three of the five patients, in whom examination of isolated nerve fibres was performed, had a number of fibres with long internodes, but inappropriately small calibres. Similar observations were published by Chopra et al. [21]. The calibre diminution suggests axonal changes, which were thought to be either secondary to schwann cell abnormalities or to arise independently. However, in the light of the present finding, they may just as well and perhaps even better be regarded as primary.

In a study of early human diabetes ultrastructural signs of primary axonal degeneration were found by Bischoff (1973) in nerve biopsies from six patients [22].

The relevance of alterations found in the peripheral nerves of streptozotocin diabetic rats to human diabetic neuropathy remains to be clearly established. At present, however, axonal changes seem to be a prominent feature in both. This suggests that streptozotocin diabetes in the rat may be accepted as a useful animal model for the elucidation of neuropathy in diabetic patients.

Acknowledgements. The author wishes to thank Felle Danvad Jensen for her technical assistance. The study was supported by Nordisk Insulinfond.

\section{References}

1. Jakobsen, J.: Axonal dwindling in carly experimental diabetes. I. A study of cross sectioned nerves. Diabetologia 12, 539-546 (1976)

2. Dyck, P. J., Gutrecht, J. A., Bastron, J. A., Karnes, W. E., Dale, A. J. D.: Histologic and teased-fiber measurements of sural nerve in disorders of lower motor and primary sensory neurons. Mayo Clin. Proc. 43, 81-123 (1968)

3. Cavanagh, J. B., Jacobs, J. M.: Some quantitative aspects of diphtheritic neuropathy. Brit. J. exp. Path. 45, 309-322 (1964)

4. Fullerton, P. M., Gilliatt, R. W., Lascelles, R. G., MorganHughes, J. A.: The relation between fibre diameter and internodal length in chronic neuropathy. J. Physiol. 178, 26P-28P (1965)

5. Sharma, A. K., Thomas, P. K.: Peripheral nerve structure and function in experimental diabetes. J. neurol. Sci. 23, 1-15 (1974)

6. Hildebrand, J., Joffroy, A., Graff, G., Coërs, C.: Neuromuscular changes with alloxan hyperglycemia. Arch. Neurol. 18, 633-641 (1968)

7. Schlaepfer, W. W., Gerritsen, G. C., Dulin, W. E.: Segmental demyelination in the distal peripheral nerves of chronically diabetic chinese hamsters. Diabetologia 10, 541-548 (1974)

8. Sencviratne, K. N., Peiris, O. A.: The effects of hypoxia on the 
excitability of the isolated peripheral nerves of alloxan diabetic rats. J. Neurol. Neurosurg. Psychiat. 32, $462-469$ (1969)

9. Sahgal, V. K., Roy, S., Ahuja, M. M. S., Singh, N.: Diabetic neuropathy. An experimental study in alloxanized rats, with special reference to insulin therapy. Acta diabet. lat. 9, 983-1006 (1972)

10. Morgan-Hughes, J. A.: Experimental diphtheritic neuropathy. A pathological and electrophysiological study. J. neurol. Sci. 7, 157-175 (1968)

11. Rasminsky, M., Sears, T. A.: Internodal conduction in undissected demyelinated nerve fibres. J. Physiol. 227, 323-350 (1972)

12. Steiness, I.: Vibratory perception in diabetics during arrested blood flow to the limb. Acta med. scand. 163, 195-205 (1959)

13. Seneviratne, K. N., Weerasuriya, A.: Nodal gap substance in diabetic nerve. J. Neurol. Neurosurg. Psychiat. 37, 502-513 (1974)

14. Anderson, M. H., Fullerton, P. M., Gilliatt, R. W., Hern, J. E. C.: Changes in the forearm associated with median nerve compression at the wrist in the guinea-pig. J. Neurol. Neurosurg. Psychiat. 33, 70-79 (1970)

15. Thomas, P. K.: The morphological basis for alterations in nerve conduction in peripheral neuropathy. Proc. roy. Soc. Med. 64, 295-298 (1971)

16. Friede, R. L.: Control of myelin formation by axon calibre. J. comp. Neurol. 144, 233-252 (1972)

17. Schmidt, R. E., Matschinsky, F. M., Godfrey, D. A., Williams, A. D., McDougal, D. B., Jr.: Fast and slow axoplasmic flow in sciatic nerve of diabetic rats. Diabetes 24, 1081-1085 (1975)
18. Reske-Nielsen, E., Lundbæk, K.: Pathological changes in the central and peripheral nervous system of young long-term diabetics. Diabetologia 4, 34-43 (1968)

19. Thomas, P. K., Lascelles, R. G.: Schwann-cell abnormalities in diabetic neuropathy. Lancet 1965 I, 1355-1357

20. Thomas, P. K., Lascelles, R. G.: The pathology of diabetic neuropathy. Quart. J. Med. 35, 489-509 (1966)

21. Chopra, J. S., Hurwitz, L. J., Montgomery, D. A. D.: The pathogenesis of sural nerve changes in diabetes mellitus. Brain 92, 391-418 (1969)

22. Bischoff, A.: Ultrastructural pathology of peripheral nervous system in early diabetes. In: Vascular and neurological changes in early diabetes. Advances in metabolic disorders, Suppl. 2 (eds. R. A. Camerini-Dávalos, H. S. Cole), pp. 441-449. New York and London: Academic Press 1973

Received: April 20, 1976, and in revised form: July 9, 1976

Dr. J. Jakobsen

Research Fellow

Second Univ. Clinic

of Internal Medicine

Kommunehospitalet

DK-8000 Aarhus C

Denmark 